Resonant optical excitation of longitudinal-optical phonons and intermixing in InAs / AlAs single quantum dots

D. Sarkar, H. P. van der Meulen, J. M. Calleja, J. M. Meyer, R. J. Haug, and K. Pierz

Citation: Appl. Phys. Lett. 92, 181909 (2008); doi: 10.1063/1.2920441

View online: https://doi.org/10.1063/1.2920441

View Table of Contents: http://aip.scitation.org/toc/apl/92/18

Published by the American Institute of Physics

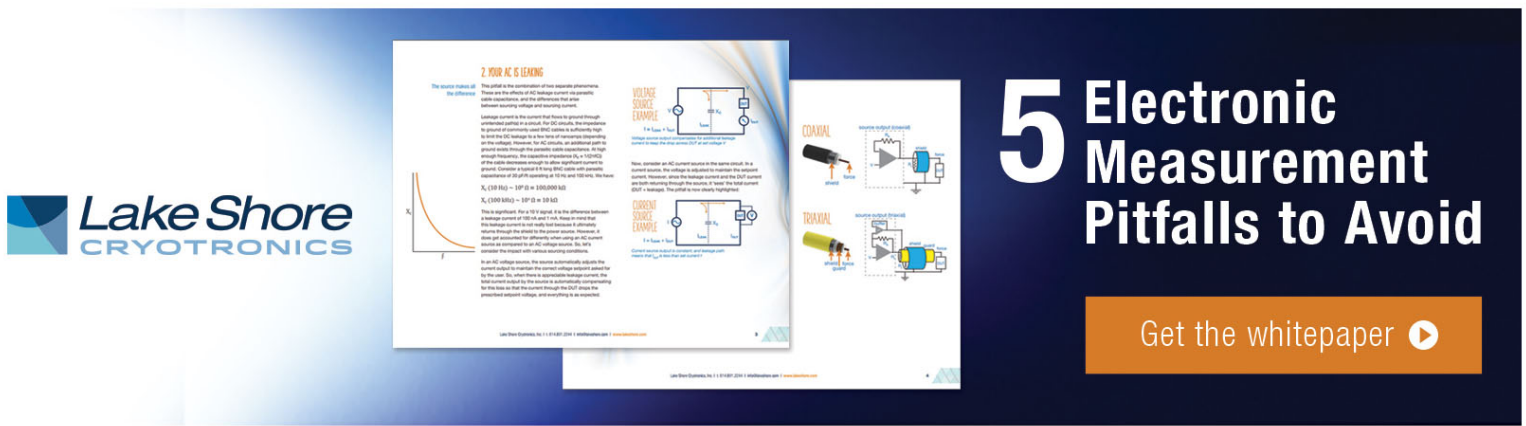




\title{
Resonant optical excitation of longitudinal-optical phonons and intermixing in InAs/AIAs single quantum dots
}

\author{
D. Sarkar, ${ }^{1, a)}$ H. P. van der Meulen, ${ }^{1}$ J. M. Calleja, ${ }^{1}$ J. M. Meyer, ${ }^{2}$ R. J. Haug, ${ }^{2}$ and \\ K. Pierz ${ }^{3}$ \\ ${ }^{1}$ Departamento Física de Materiales, Universidad Autónoma de Madrid, E-28049 Madrid, Spain \\ ${ }^{2}$ Institut für Festkörperphysik, Leibniz. Universität Hannover, D-30167 Hannover, Germany \\ ${ }^{3}$ Physikalisch-Technische Bundesanstalt Braunschweig, D-38116 Braunschweig, Germany
}

(Received 24 October 2007; accepted 16 April 2008; published online 9 May 2008)

Excitations of a single InAs/AlAs self-assembled quantum dot were investigated by photoluminescence excitation spectroscopy. Resonant absorption by longitudinal-optical (LO) phonons of the quantum dot and the barriers is observed. In particular, a resonance at $41 \mathrm{meV}$ is attributed to the AlAs-like mode of InAlAs with low Al content. Our results represent a direct optical measure of the intermixing (estimated below 10\%) in a single quantum dot. They also demonstrate that all LO phonons of this mixed quantum dot system couple to the exciton states. (C) 2008 American Institute of Physics. [DOI: 10.1063/1.2920441]

The study of the properties of individual semiconductor quantum dots ${ }^{1-3}$ (QDs) is of great interest for application in classical and quantum ${ }^{4}$ light emitters and detectors as well as for the implementation of quantum information processing systems. ${ }^{5}$ Although these applications require very different conceptual approaches toward devices, the understanding of the scattering mechanisms is of fundamental importance in all cases. Indeed, they are crucial to obtain fast carrier capture, efficient carrier relaxation, and long recombination lifetimes. In self-assembled QDs (SAQDs), the strong excitonphonon coupling leads to the formation of polaron states, ${ }^{6-10}$ whose decay into acoustic phonons is a major contribution to relaxation. Phonons in SAQDs can be studied by optical techniques, as reported in photoluminescence (PL), PL excitation (PLE), and Raman measurements. ${ }^{11-14}$

In this work, we report PL and PLE data on InAs/AlAs SAQDs, where strong confinement results in a single electron state localized in the QD. In these QDs, carrier relaxation would be expected only by direct scattering from the continuum of extended excited states to the ground confined state. However, we show that PL emission is enhanced by resonant excitation involving different longitudinal-optical (LO) phonons in the system. One of these phonons is the high-energy mode of AlInAs for low Al concentration. This result demonstrates the presence of $\mathrm{Al}$ intermixing in InAs QDs, detected by means of optical measurements. Our results also show that both intra dot and barrier optical phonons are coupled to the excitonic states and contribute to their relaxation.

A single layer of InAs/AlAs QDs was grown by molecular beam epitaxy on GaAs (100) substrates, as described in Ref. 15. Typical values are QD densities of $10^{10} \mathrm{~cm}^{-2}$, dot diameters between 25 and $30 \mathrm{~nm}$, and dot heights around $3 \mathrm{~nm}$. The samples were covered by an aluminium mask with squared apertures of sizes ranging from 10 to $0.2 \mu \mathrm{m}$ fabricated by electron beam lithography. The smaller apertures contain few dots, allowing for detection of single QD luminescence. Considering the high confinement due to the

\footnotetext{
${ }^{\text {a) }}$ Author to whom correspondence should be addressed. Electronic mail:
} dipankar.sarkar@uam.es.
AlAs barriers and the small QD size, only one electron state and up to two heavy-hole (HH) states are expected to be localized in the QD. The optical measurements were performed exciting with a Ti-sapphire laser using a microscope setup with a spot size of $5 \mu \mathrm{m}$. A double grating spectrometer with a charge-coupled device detector was used for detection. All the measurements were taken at $9 \mathrm{~K}$.

The PL spectra of a single QD emitting at $1.565 \mathrm{eV}$ is shown in Fig. 1 for resonant $(1.643 \mathrm{eV})$ and nonresonant $(2.410 \mathrm{eV})$ excitation. The low-energy peaks in both spectra correspond to emissions of the exciton $\left(X^{0}\right)$, biexciton $(X X)$ and most probably trion $\left(X^{+}\right)$of a single QD, together with an exciton $X^{0}$ (QD2) belonging to a different dot. Peaks of the same QD were identified by their jitter pattern, polarization, and power dependence. ${ }^{16}$ The peaks at higher energies probably belong to different QDs except the ones around $1.597 \mathrm{eV}$, which also appear in the PLE spectrum shown in

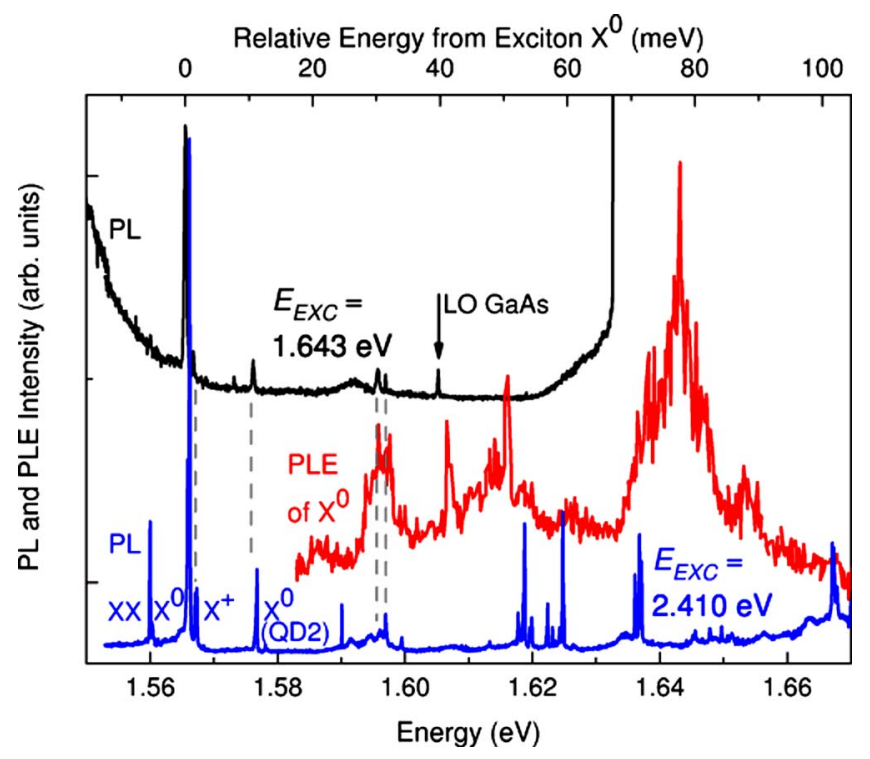

FIG. 1. (Color online) Resonantly (1.64 eV, black) and nonresonantly $\left(2.41 \mathrm{eV}\right.$, blue) excited PL. The marked peaks are the exciton $X^{0}$, biexciton $X X$, and probably trion $X^{+}$of the same QD. The PLE spectrum of $X^{0}$ (red curve) shows phonon resonances at $31,41,51$, and $75 \mathrm{meV}$. 


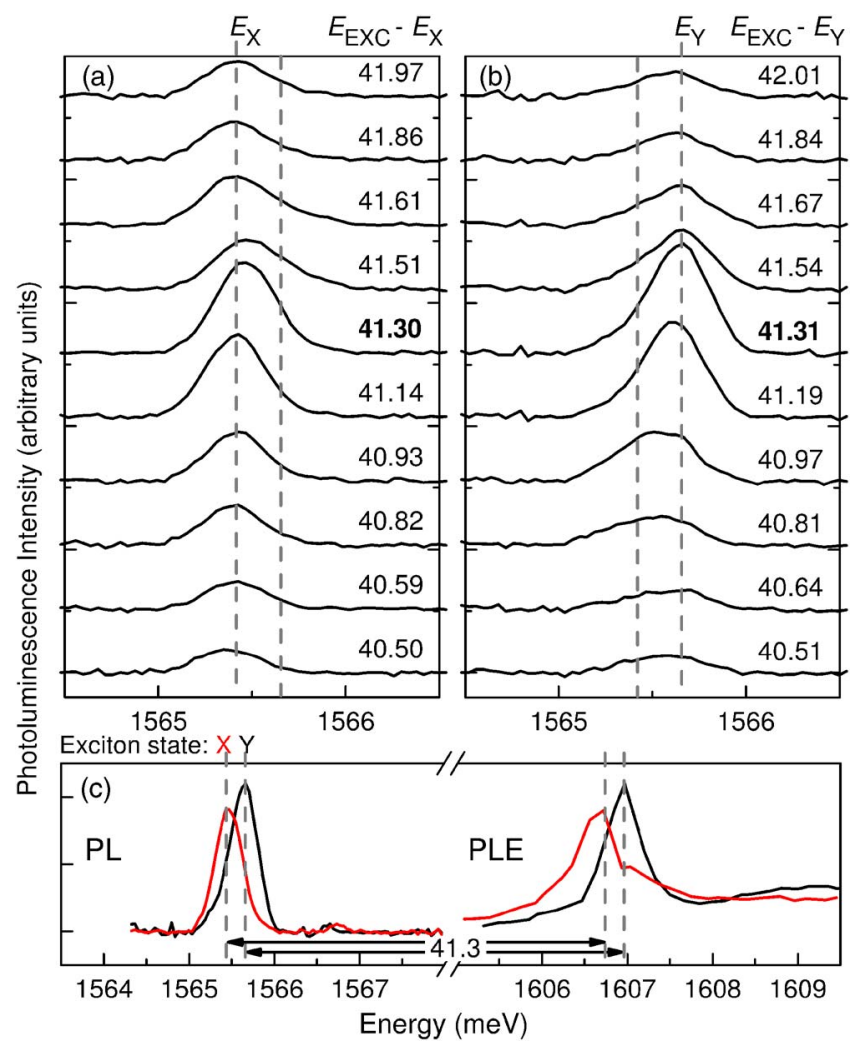

FIG. 2. (Color online) Polarization resolved PL spectra for varying excitation energies. Left and right panel show the linearly counterpolarized exciton states $X$ and $Y$, respectively. Bottom panel: the fine structure split exciton lines $X$ and $Y$ resonate for excitation at $41.3 \mathrm{meV}$ above the respective emission lines.

Fig. 1. The PLE spectrum exhibits two broad resonances at 31 and $75 \mathrm{meV}$, as well as two narrow ones at 41.3 and $51 \mathrm{meV}$. All these resonances are attributed to phonon assisted absorption or Raman scattering processes.

The PL peaks at 31 and $32 \mathrm{meV}$ (relative energy, upper scale) in the PL spectra coincide with the broad PLE resonance. In accordance to previous work, we attribute them, likewise the PLE peak at $75 \mathrm{meV}$, to polaron states $7,17-20$ formed by the QD InAs $\mathrm{LO}^{11,12,14,21,22}$ and perhaps interface (IF) phonons. ${ }^{23,24}$

In the following, we concentrate on the resonance at $41.3 \mathrm{meV}$. Figure 2 depicts a series of PL spectra obtained for excitation energies varying roughly in $0.17 \mathrm{meV}$ steps from 40.5 to $42.0 \mathrm{meV}$ above the fine-structure split exciton transitions around $1.5655 \mathrm{eV}$. Left and right panels correspond to polarization emissions parallel to the crystallographic directions $(110)$ and $(1-10)$ (labeled $X$ and $Y$ ), respectively. In each panel, only the corresponding component of the split exciton $(X, Y)$ is observed. As the laser energy is increased, both exciton transitions and the trion $X^{+}$at $1.5668 \mathrm{eV}$ experience successive enhancements in the PL intensity. The bottom panel of Fig. 2 shows the PL and PLE spectra of transitions $X$ and $Y$. One sees that the resonance at $41.3 \mathrm{meV}$ is common for both $X, Y$ exciton components and that the fine-structure splitting is well resolved in PLE. The unpolarized PL intensity is plotted in Fig. 3 as color scale plot versus detection and excitation energy. Horizontal and vertical cuts correspond to PL and PLE spectra, respectively. The dotted line indicates an energy of $41.3 \mathrm{meV}$ below the excitation energy. The resonances of the split exciton and the trion (clearly seen in this plot) are observed when the exci-

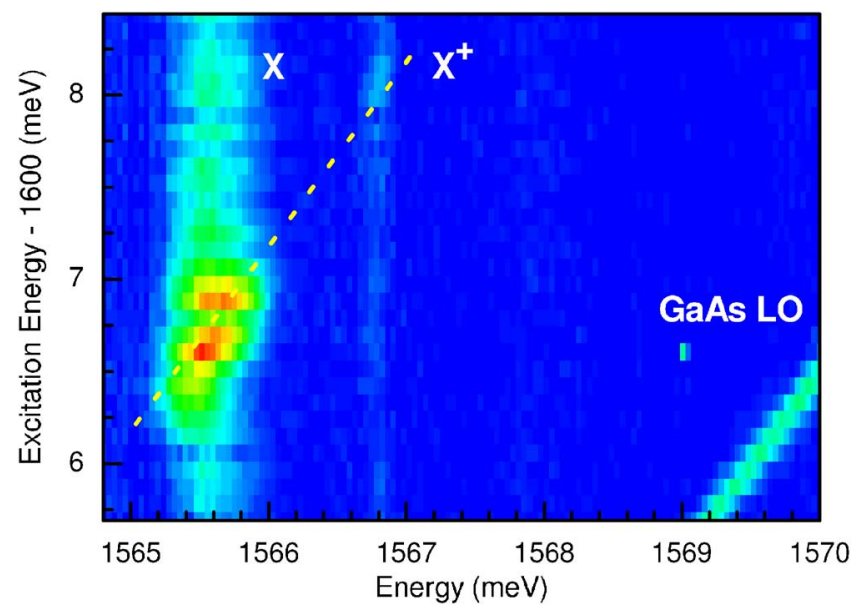

FIG. 3. (Color online) Intensity scale plot of the PL versus excitation and detection energy. The dotted line is $41.3 \mathrm{meV}$ below the laser energy. Notice that also the trion $X^{+}$resonates at the same excess energy.

tation and emission energies differ by $41.3 \mathrm{meV}$. The emission parallel to the dotted line at the right hand side of Fig. 3 is the LO phonon of the GaAs substrate. We exclude excited states as the origin of the resonances at $40 \mathrm{meV}$ (Refs. 11 and 12) due to the high AIAs barriers. We attribute the excitation at $41.3 \mathrm{meV}$ to a phonon that "moves" parallel to the excitation energy, as the bulk GaAs-LO one, and successively resonates with the three PL lines of the QD. One might discuss whether the resonance is due to phonon assisted absorption or to resonant Raman scattering. In the latter case, one should resolve the phonon peak from the PL emission by slightly detuning the excitation energy from the resonance. However, the small width of the resonance ( $0.4 \mathrm{meV}$, comparable to the phonon line width) makes this impossible in the present case, and both processes become indistinguishable. The relevant phonons of InAs/AlAs QDs are LO, TO and IF modes, whose energy ranges in unstrained materials are 27-30 meV for InAs and 45-50 meV for AlAs, respectively. For fully strained AlAs (matching the InAs lattice parameter), the range is $41-43 \mathrm{meV}$ (Ref. 23) while the LO frequency for strained InAs is around $32 \mathrm{meV} .^{21,22}$ The only candidate for the $41.3 \mathrm{meV}$ phonon would be the TO mode of fully strained AlAs. However, this is unlikely, as the coupling of TO phonons to excitons is much weaker than the LO one and AlAs barriers are expected to be essentially unstrained. These arguments also rule out the AlAs-like IF modes. ${ }^{24}$ Instead, we attribute the single QD excitation responsible for the PLE resonance at $41.3 \mathrm{meV}$ to the AlAs-like LO mode of the InAlAs ternary compound. This implies an $\mathrm{Al}$ concentration of less than $10 \%$ in our QDs. ${ }^{25}$ The occurrence of Al-In intermixing in similar QDs was evidenced by tunnelling microscopy. ${ }^{26}$ This small Al concentration does not change the InAs-like phonon frequency, as it is essentially independent on composition. ${ }^{25}$ Finally, the resonance at $51 \mathrm{meV}$ corresponds to the unstrained AlAs LO phonon of the QD barriers. It can couple to the exciton due to the wave function leaking into the barrier. Barrier phonons have been widely reported for QDs embedded in GaAs. ${ }^{11-14}$

To get a deeper insight into the scattering mechanism, we analyze the PL polarization properties of the $41.3 \mathrm{meV}$ resonance. In Fig. 4, the four polarization configurations are plotted for excitation near the $\mathrm{Y}$ exciton resonance. The 


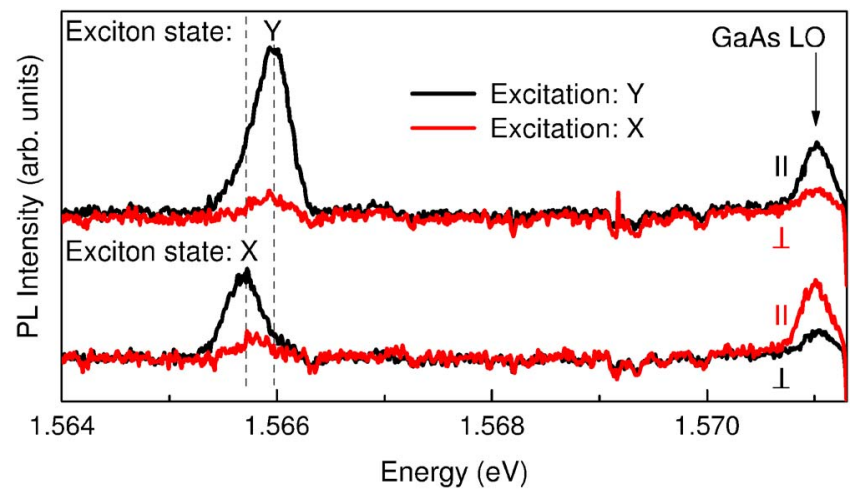

FIG. 4. (Color online) Polarization resolved PL spectra for excitation near the $Y$ exciton resonance. Both $X, Y$ exciton states can mostly be observed for $Y$ polarized excitation, indicating a doubly resonant process.

emission is much more intense for $Y$ than for $X$ polarized excitation, regardless of the emission polarization. This difference points to a broken symmetry of the QD states in the (110) and $(1-10)$ directions, probably due to the anisotropy of the piezoelectric potential. As this anisotropy can split the excited-hole states of up to several tens of $\mathrm{meV}^{22}$ we can speculate that one of the split excited-hole states is situated closer to $41 \mathrm{meV}$, above the ground $\mathrm{HH}$ state than its counterpart. This would favor the excitation in a doubly resonant process for one polarization $(Y)$, as observed. In the relaxation process, scattering by the $\mathrm{LO}$ phonons results in ground state excitons with both $X, Y$ symmetries.

In summary, we studied the resonant excitation of the PL emission in a single InAs/AlAs QD. We find resonances corresponding to all the LO phonons of the QD system (InAs-like and AlAs-like of the QD, and AlAs-LO of the barrier). In particular we associate the $41.3 \mathrm{meV}$ resonance to the AlAs-like LO phonon of InAlAs with small Al concentration. This constitutes a direct optical determination of the intermixing in a single QD. All of the observed phonons couple to the QD electronic transitions and can thus contribute to carrier relaxation.

This work has been supported by research contracts of the Spanish Ministry of Education (MEC MAT200501388, NAN2004-09109-C04-04, TEC2004-05260-C02-02, Consolider CSD 2006-19) and the Community of Madrid (CAM S-0505-ESP-0200).
${ }^{1}$ A. Zrenner, L. V. Butov, M. Hagn, G. Abstreiter, G. Böhm, and G. Weimann, Phys. Rev. Lett. 72, 3382 (1994).

${ }^{2}$ J. Y. Marzin, J. M. Gérard, A. Izrael, D. Barrier, and G. Bastard, Phys. Rev. Lett. 73, 716 (1994)

${ }^{3}$ D. Gammon, E. Snow, B. Shanabrook, D. Katzer, and D. Park, Phys. Rev. Lett. 76, 3005 (1996).

${ }^{4}$ O. Benson, C. Santori, M. Pelton, and Y. Yamamoto, Phys. Rev. Lett. 84, 2513 (2000).

${ }^{5}$ X. Li, Y. Wu, D. Steel, D. Gammon, T. H. Stievater, D. S. Katzer, D. Park, C. Piermarocchi, and L. J. Sham, Science 301, 809 (2003).

${ }^{6}$ X.-Q. Li, H. Nakayama, and Y. Arakawa, Phys. Rev. B 59, 5069 (1999).

${ }^{7}$ T. Inoshita and H. Sakaki, Phys. Rev. B 56, R4355 (1997).

${ }^{8}$ V. M. Fomin, V. N. Gladilin, J. T. Devreese, E. P. Pokatilov, S. N. Balaban, and S. N. Klimin, Phys. Rev. B 57, 2415 (1998).

${ }^{9}$ O. Verzelen, R. Ferreira, and G. Bastard, Phys. Rev. Lett. 88, 146803 (2002).

${ }^{10}$ R. P. Miranda, M. I. Vasilevskiy, and C. Trallero-Giner, Phys. Rev. B 74, 115317 (2006).

${ }^{11}$ F. Findeis, A. Zrenner, G. Böhm, and G. Abstreiter, Phys. Rev. B 61, R10579 (2000).

${ }^{12}$ R. Oulton, J. J. Finley, A. I. Tartakovskii, D. J. Mowbray, M. S. Skolnick, M. Hopkinson, A. Vasanelli, R. Ferreira, and G. Bastard, Phys. Rev. B 68, 235301 (2003)

${ }^{13}$ Y. Toda, O. Moriwaki, M. Nishioka, and Y. Arakawa, Phys. Rev. Lett. 82, 4114 (1999).

${ }^{14}$ A. Lemaître, A. D. Ashmore, J. J. Finley, D. J. Mowbray, M. S. Skolnick, M. Hopkinson and T. F. Krauss, Phys. Rev. B 63, 161309 (2001).

${ }^{15}$ Z. Ma, K. Pierz, F. J. Ahlers, U. F. Keyser, and R. J. Haug, in Proceedings of the 26th International Conference on the Physics of Semiconductors, IOP Conf. Proc. No. 171, edited by R. A. Long and J. H. Davis (Institute of Physics, Bristol, 2002).

${ }^{16}$ D. Sarkar, H. P. van der Meulen, J. M. Calleja, J. M. Becker, R. J. Haug, and K. Pierz, J. Appl. Phys. 100, 023109 (2006).

${ }^{17}$ D. Sarkar, H. P. van der Meulen, J. M. Calleja, J. M. Becker, R. J. Haug, and K. Pierz, Phys. Rev. B 71, 081302 (2005).

${ }^{18}$ S. Hameau, Y. Guldner, O. Verzelen, R. Ferreira, G. Bastard, J. Zeman, A. Lematre, and J. M. Grard, Phys. Rev. Lett. 83, 4152 (1999).

${ }^{19}$ E. A. Zibik, L. R. Wilson, R. P. Green, G. Bastard, R. Ferreira, P. J. Phillips, D. A. Carder, J.-P. R. Wells, J. W. Cockburn, M. S. Skolnick, M. J. Steer, and M. Hopkinson., Phys. Rev. B 70, 161305 (2004).

${ }^{20}$ B. Aslan, H. C. Liu, M. Korkusinski, P. Hawrylak, and D. J. Lockwood, Phys. Rev. B 73, 233311 (2006).

${ }^{21}$ R. Heitz, M. Veit, N. Ledentsov, A. Hoffmann, D. Bimberg, V. Ustinov, P. Kopev, and Z. I. Alferov, Phys. Rev. B 56, 10435 (1997).

${ }^{22}$ M. Grundmann, O. Stier, and D. Bimberg, Phys. Rev. B 52, 11969 (1995).

${ }^{23}$ D. A. Tenne, V. A. Haisler, A. I. Toropov, A. K. Bakarov, A. K. Gutakovsky, D. R. T. Zahn, and A. P. Shebanin, Phys. Rev. B 61, 13785 (2000).

${ }^{24}$ A. G. Milekhin, A. I. Toropov, A. K. Bakarov, D. A. Tenne, G. Zanelatto, J. C. Galzerani, S. Schulze, and D. R. T. Zahn, Phys. Rev. B 70, 085314 (2004).

${ }^{25}$ S. Emura, T. Nakagawa, S. Gonda, and S. Shimizu, J. Appl. Phys. 62, 4632 (1987).

${ }^{26}$ P. Offermans, P. M. Koenraad, J. H. Wolter, K. Pierz, M. Roy, and P. A. Maksym, Phys. Rev. B 72, 165332 (2005). 